\title{
The Fried Frailty Criteria as Inclusion Criteria for a Randomized Controlled Trial: Personal Experience and Literature Review
}

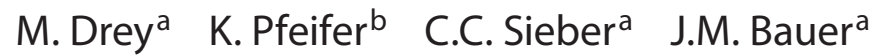 \\ ${ }^{\mathrm{a}}$ Department of Geriatric Medicine, and ${ }^{\mathrm{b}}$ Institute of Sport Science and Sport, University of Erlangen-Nuremberg, \\ Nuremberg, Germany
}

\section{Key Words}

Fried criteria • Frailty · Applicability, frailty criteria •

Recruitment

\begin{abstract}
Background: Among current operational definitions of frailty, the criteria proposed by Fried and colleagues have attracted great scientific interest. However, these criteria have usually been applied in epidemiological and only rarely in interventional studies. Objective: The present paper aims at testing the applicability of the Fried frailty criteria in the context of the recruitment process of a randomized controlled trial in prefrail older persons, and it discusses the respective scientific literature. Methods: Recruitment was promoted by newspaper articles as well as by targeted mail to customers of a local health insurance company and to recently treated patients of a geriatric day clinic. Furthermore, presentations were given in assisted living facilities. Potential candidates were screened for prefrailty, i.e. to see whether they met 1 or 2 of the Fried criteria (weight loss, handgrip strength, gait speed, exhaustion, physical activity). Results: A total of 298 people were screened. Among them 181 were not frail, 116 were prefrail and 1 was diagnosed as frail. The most prevalent criterion was exhaustion ( $24 \%$ of those screened). The second most prevalent criterion was low handgrip strength
\end{abstract}

(20\%). Low gait speed (8\%), low physical activity (2\%) and weight loss (2\%) had a lower prevalence. According to the Geriatric Depression Scale, $14 \%$ of those who met the criterion 'exhaustion' were depressed. With regard to the Minnesota Leisure Time Physical Activity Questionnaire, used for the evaluation of 'physical activity', only 3 activities among the 18 selected by Fried were applicable to our cohort. Conclusions: Under the study conditions, good applicability of the Fried criteria was observed. Nevertheless, further refinement may be expedient in several criteria, especially exhaustion and physical activity, to enhance clinical usefulness. It may be helpful to adapt the cutoffs when applying the criteria to a European population. Copyright $\odot 2010$ S. Karger AG, Basel

\section{Introduction}

The first article found when searching PubMed using the MeSH term 'frail elderly' was published in 1953. Considering the frequency of publications in the ensuing years, frailty created only little scientific interest until the late 1980s. At the beginning of the 1990s, the number of papers published on frailty escalated, with nearly 5,500 articles listed on PubMed.

The frailty syndrome has been defined in different ways, and several diagnostic tools have been developed.

\section{KARGER \\ Fax +41613061234 \\ E-Mail karger@karger.ch}

www.karger.com
(C) 2010 S. Karger AG, Basel

0304-324X/11/0571-0011\$38.00/0

Accessible online at:

www.karger.com/ger 
Nevertheless, no general consensus has been reached with regard to its diagnosis $[1,2]$. At the Second International Working Meeting on Frailty and Aging, held in Montreal in March 2006 [3], it was generally agreed that the frailty syndrome is characterized by an increased vulnerability to external and internal stressors due to impairments in multiple, interrelated physiological systems. This vulnerability leads to a decline in homeostatic reserve and resilience, and implies an increased risk for multiple adverse health-related outcomes. Based on these core features, two main phenotypes of frailty were described [4], one of which is characterized by a multi-domain concept. In addition to physical components, this concept addresses cognition, mood, sensory impairments, chronic diseases, disabilities and social conditions $[5,6]$. The second concept, which may be regarded as one-dimensional, describes a physical phenotype of frailty, which was defined by Fried et al. [7]. Its criteria include shrinking (weight loss), weakness (handgrip strength), exhaustion or poor endurance (self-reported exhaustion), slowness (gait speed), and low activity (kilocalories expended per week). In this context, clusters of physical impairments shape frailty, and non-physical components are not considered.

The Fried criteria have been applied in large epidemiological studies, especially in the United States, Canada and Europe [8-11]. In this context, some of the criteria have been slightly modified. For widespread acceptance of the frailty concept among clinicians, it is essential that its diagnosis is clinically feasible and that it bears clinical significance with regard to specific interventions and prognosis $[4,12]$.

We applied the Fried criteria to define a study cohort for an intervention study in prefrail older individuals (NCT00783159). In expert opinion, prefrail individuals are regarded as a suitable target group for intervention studies, which aim to improve the frailty status and prevent further functional decline [4]. The intervention in the present study consisted of different training modes.

In this paper, we present our results on the applicability of the Fried criteria in the recruitment process for the aforementioned study. Applicability, cutoffs and the validity of the Fried criteria are discussed in the context of the scientific literature.

\section{Subjects and Methods}

\section{Inclusion and Exclusion Criteria}

The original Fried criteria that were applied in the present study are given in table 1 . The frailty score of Fried comprises a possible range from 0 to 5 . An older person ( $>65$ years) is consid- ered frail if three or more criteria are assessed as positive. Individuals who do not meet any of the criteria listed in table 1 are regarded as not being frail, whereas people with one or two positive criteria are considered to be prefrail. This categorization correlates with adverse health outcome, as has been shown by Fried et al. [7]. For the present randomized controlled intervention trial, we recruited almost exclusively community-dwelling prefrail individuals over the age of 65 . This study aims to examine the effects of two different strength training modalities (resistance training vs. power training) on functionality. After frailty screening, participants with depression (GDS >5), dementia (MMSE $<25$ ), BMI $>35$, intake of immunosuppressive drugs, a history of kidney stones, sarcoidosis, plasmocytoma, chronic obstructive pulmonary disease, inflammatory bowel disease, angina pectoris, a history of cancer, arthritis, and current attendance of muscle training were excluded.

\section{Recruitment of Study Cohort}

Four different strategies were applied in the recruitment process. First, a press conference was held to explain the modalities and objectives of the study. Two articles were published in local newspapers to introduce the study to the public. Older people who were interested in participating in the study were asked to call the study center. Potentially eligible people were invited to the study center to be screened.

Second, cooperation was established with a local health insurance company. With the help of this company, personally addressed letters presenting information on the study were sent to individuals over the age of 65 who lived close to the study center. The recipients were asked to complete a return form. After checking the forms, eligible participants were invited for screening.

The third strategy focused on assisted living facilities. Information flyers were distributed to the residents of 10 local institutions. Those interested in participating in the study were asked to call the study center. Eligible candidates were then invited to our institution.

The final strategy involved the database of the Nuremberg geriatric day clinic. Patients who had received treatment the previous year were sent personally addressed letters along with a return form. Potentially eligible participants were invited for screening.

The study was approved by the Medical Ethics Committee of the University Erlangen-Nuremberg, and informed consent was obtained from all participants before personal data were collected and recorded.

\section{Results}

Figure 1 illustrates the recruitment process. A total of 116 prefrail individuals were identified. The most prevalent criterion was exhaustion (24\%), followed by low handgrip strength (20\%). Low gait speed (8\%), weight loss (2\%) and low physical activity (2\%) were much less frequently positive (fig. 2). Fourteen percent of those who scored positive for the criterion 'exhaustion' were probably depressed according to the Geriatric Depression Scale. With regard to the Minnesota Leisure Time Physi- 


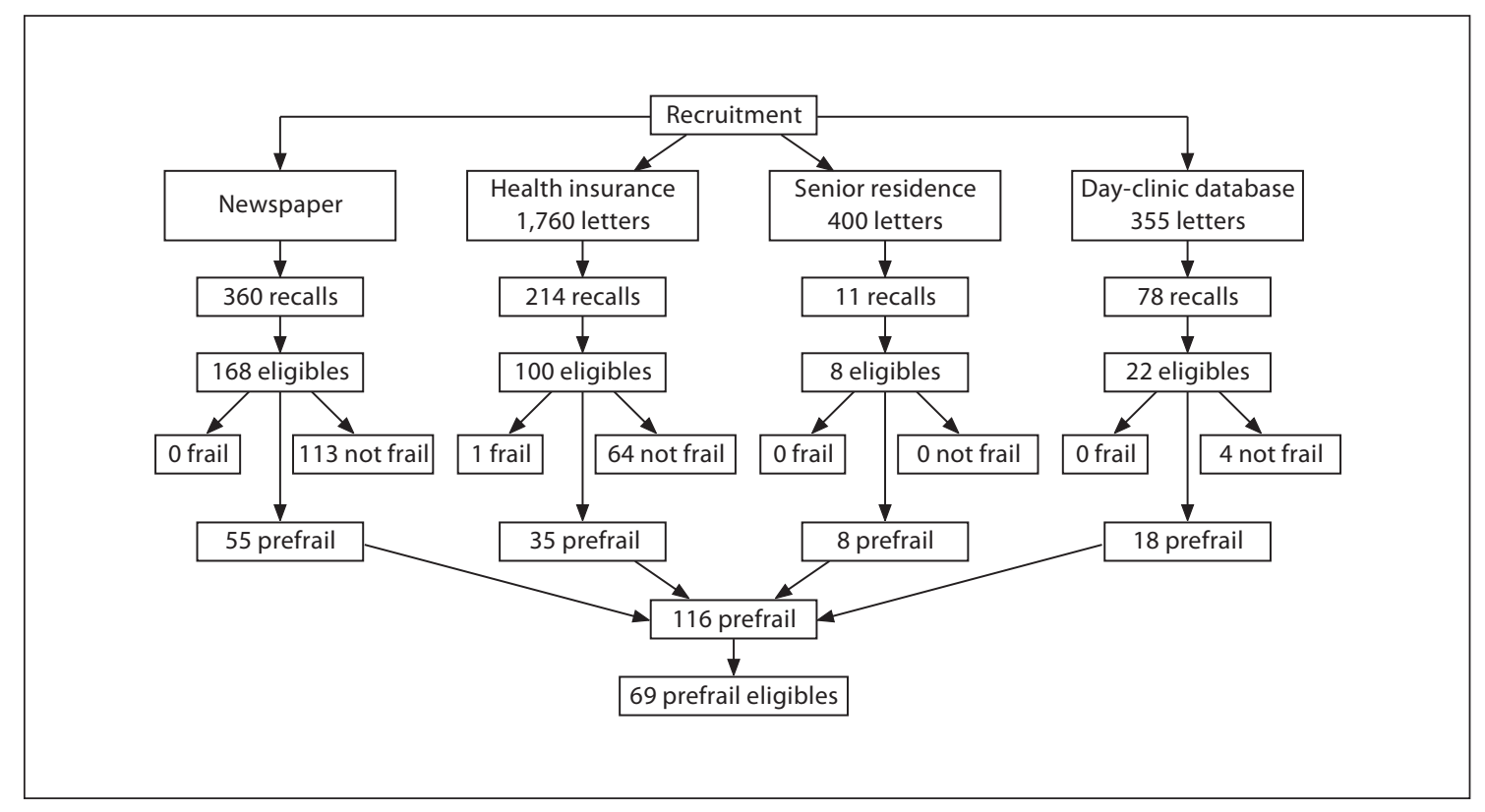

Fig. 1. Recruitment process and frailty screening in eligible persons.

Table 1. Criteria for the definition of frailty developed by Fried et al. [7]

\begin{tabular}{|c|c|c|c|c|}
\hline $\begin{array}{l}\text { Shrinking, } \\
\text { i.e. weight loss }\end{array}$ & \multicolumn{4}{|c|}{$\begin{array}{l}\text { Unintentional loss of } 4.5 \mathrm{~kg} \text { ( } 10 \mathrm{lbs} \text { ) in the year before the current evaluation or unintentional weight loss } \\
\text { of at least } 5 \% \text { of the previous year's body weight }\end{array}$} \\
\hline \multirow[t]{4}{*}{$\begin{array}{l}\text { Weakness, } \\
\text { i.e. low handgrip strength }\end{array}$} & \multicolumn{4}{|c|}{$\begin{array}{l}\text { Grip strength of the dominant hand (mean of three measurements), using a Jamar hand-held } \\
\text { dynamometer: }\end{array}$} \\
\hline & $\mathrm{BMI} / \mathrm{male}$ & Cutoff (kg) & BMI/female & Cutoff (kg) \\
\hline & $\leq 24$ & $\leq 29$ & $\leq 23$ & $\leq 17$ \\
\hline & $24-26$ & $\leq 30$ & $23-26$ & $\leq 17.3$ \\
\hline
\end{tabular}

Poor endurance, i.e. self-reported exhaustion
Evaluation of two statements of the CES-D scale:

(a) I felt that everything I did was an effort

(b) I could not get going

Criterion positive if at least one condition is present for 3 days or more during the last week.

\begin{tabular}{lllll}
\hline Slowness, i.e. low gait & Cutoff for time to walk $4.57 \mathrm{~m}(15 \mathrm{ft})$ at usual pace (static protocol): & \\
speed & Height/male $(\mathrm{cm})$ & Cutoff $(\mathrm{s})$ & Height/female $(\mathrm{cm})$ & Cutoff $(\mathrm{s})$ \\
& $\leq 173$ & $\geq 7(0.65 \mathrm{~m} / \mathrm{s})$ & $\leq 159$ & $\geq 7(0.65 \mathrm{~m} / \mathrm{s})$ \\
& $>173$ & $\geq 6(0.76 \mathrm{~m} / \mathrm{s})$ & $>159$ & $\geq 6(0.76 \mathrm{~m} / \mathrm{s})$
\end{tabular}

Low activity, i.e. reduced Evaluation of 18 activities from the Minnesota Leisure Time Physical Activity Questionnaire. Time spent energy consumption on each activity is to be recorded in minutes for the last 2 weeks and then multiplied by an activity score. Half of the total of all activities, expressed in kilocalories per week, represents the physical activity of an evaluated individual per week. The physical activity criterion is positive if physical activity per week is for: Male Female $<383 \mathrm{kcal} /$ week $\quad<270 \mathrm{kcal} /$ week

CES-D = Center for Epidemiological Studies Depression. 


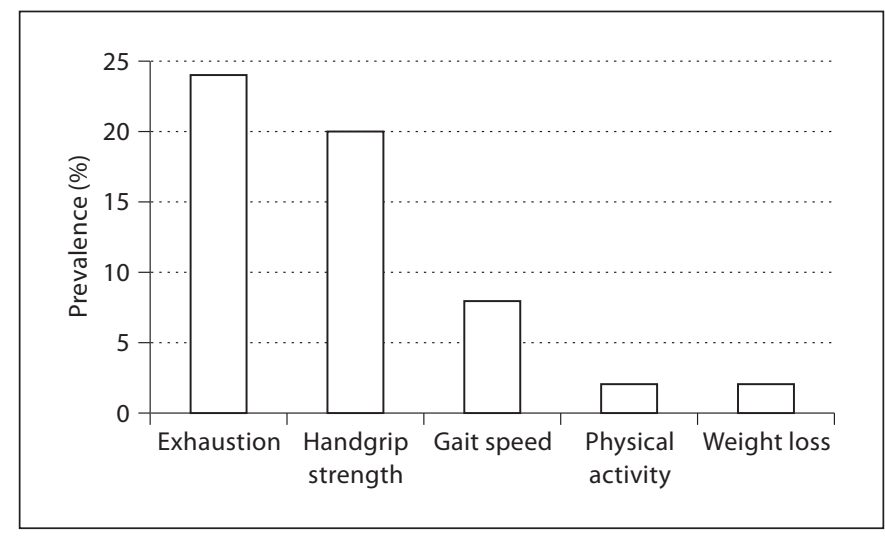

Fig. 2. Prevalence of frailty criteria among screened participants.

cal Activity Questionnaire (MLTPAQ), used for the evaluation of 'physical activity', only three activities (walking, biking, swimming) among the 18 selected by Fried were relevant in our study cohort. Finally, only 69 of the 116 prefrail individuals were included in the randomized controlled intervention trial. Most of those who decided not to join the project did so due to the inconvenience of reaching the study center for training. A smaller number had to be excluded due to probable depression or because they had a BMI of $>35$.

\section{Discussion}

In our cohort of potentially eligible study participants, the cutoffs for weight loss and gait speed from Fried et al. [7] appeared to be rather strict as only a small number of screened individuals was positive for these two criteria. Furthermore, the spectrum of physical activity in our cohort was not adequately represented by the MLTPAQ. Considering the significant overlap of depressive traits with subjective exhaustion, the validity of the respective criterion may be questioned. When applying them to European populations, it may be expedient to adapt the cutoffs set by Fried. In the following text, the above observations will be discussed with regard to the current scientific literature.

For the present study, we exclusively recruited community-dwelling prefrail older persons who lived conveniently close to the study center and who were able to attend training sessions on a regular basis. Our sample will therefore not be representative for the German population beyond the age of 65 in general. Our observations will provide some insight into relevant differences between the Cardiovascular Health Study (CHS) cohort and a cohort of German community-living older persons. Moreover, we will discuss the prevalence of each Fried criterion in our study cohort in relation to the results of 4 large epidemiologic studies in which the Fried criteria were also applied. These epidemiologic studies are the Women's Health and Aging Study (WHAS) [8], the Invecciare in Chianti Study (InCHIANTI Study) [9], the Osteoporotic Fractures in Men Study (MrOS) [10] and the Survey of Health, Aging and Retirement in Europe (SHARE) [11]. The criteria applied, the prevalence of frailty, and the references are shown in table 2.

The cutoffs used in the Fried criteria were based on US data from the CHS. For handgrip strength, gait speed and physical activity, values in the lowest quintile were defined as indicating frailty. We would like to stress that only one or two criteria need to be positive for prefrailty to be diagnosed. For this reason, each single criterion should demonstrate high validity with regard to this diagnosis.

In our cohort, the prevalence for the weight loss criterion according to Fried was $2 \%$. This criterion implies an unintentional loss of $>4.5 \mathrm{~kg}$ in the course of the last year or an unintentional weight loss of at least $5 \%$ of the body weight measured a year before. When scrutinizing the four aforementioned studies, it must be noted that the weight loss criterion was modified in three of them. Only the InCHIANTI Study applied the Fried criterion in its original form. In the WHAS, this criterion scored positive if there was a weight loss of at least $10 \%$, with the reference being the individual's weight at the age of 60 or, alternatively, if the actual BMI was $<18.5 \mathrm{~kg} / \mathrm{m}^{2}$. In the SHARE Study, the weight loss criterion was met by reporting a 'diminution in desire for food' in response to the question, 'What has your appetite been like' or, in the case of an unusable response to this question, by responding 'less' to the following: 'So have you been eating more or less than usual?' In the MrOS cohort, the authors intended this criterion to reflect sarcopenia, as this condition is closely linked to the physical concept of frailty. Appendicular skeletal muscle mass (ASM), measured by dual energy X-ray absorptiometry (DEXA), was regressed in linear models on height $(\mathrm{cm})$ and total body fat $(\mathrm{kg})$. The difference between actual ASM and expected ASM according to the linear regression model was then used to quantify sarcopenia for each participant. A negative residual indicated that a participant had a lower than expected ASM for their height and fat mass. The participants with residuals comprising the lowest $20 \%$ were con- 
Table 2. Four epidemiologic studies using modified Fried criteria

\begin{tabular}{|c|c|c|c|c|}
\hline Study & WHAS [8] & InCHIANTI [9] & $\operatorname{MrOS}[10]$ & SHARE $^{1}[11]$ \\
\hline Participants & 786 우 & 827 잉 & 5,993 \&े & $933+18$ \\
\hline Frailty & 11.3 & 6.5 & 4 & 12.1 \\
\hline Prefrailty & 43.8 & 37.8 & 40 & 34.6 \\
\hline No frailty & 44.9 & 55.7 & 56 & 53.3 \\
\hline $\begin{array}{l}\text { Handgrip } \\
\text { (prevalence) }\end{array}$ & $\begin{array}{l}\text { Like CHS } \\
(20.8 \%)\end{array}$ & $\begin{array}{l}\text { Like CHS, but cohort } \\
\text { based }(17.5 \%)\end{array}$ & $\begin{array}{l}\text { Like CHS } \\
(13 \%)\end{array}$ & $\begin{array}{l}\text { Like CHS } \\
(15.1 \%)\end{array}$ \\
\hline $\begin{array}{l}\text { Exhaustion } \\
\text { (prevalence) }\end{array}$ & $\begin{array}{l}\text { Felt unusually tired/weak } \\
\text { last week }(14.1 \%)\end{array}$ & $\begin{array}{l}\text { Like CHS } \\
(16.6 \%)\end{array}$ & $\begin{array}{l}\text { Lot of energy } \\
\text { last month }(8 \%)\end{array}$ & $\begin{array}{l}\text { Too little energy to do } \\
\text { things last month }(30.0 \%)\end{array}$ \\
\hline
\end{tabular}

DXA = Dual energy X-ray absorptiometry; PASE = Physical Activity Scale for the Elderly; WHAS = Women's Health and Aging Study; InCHIANTI = Invecciare in Chianti Study; MrOS = Osteoporotic Fractures in Men Study; SHARE = Survey of Health, Aging and Retirement in Europe; CHS = Cardiovascular Health Study.

${ }^{1}$ Data for Germany and participants above the age of 65 .

sidered positive for this criterion. These modifications have probably influenced the prevalence of the weight loss criterion in the respective study populations. Only the InCHIANTI Study, which applied the original weight loss criterion, showed prevalence close to the result of the CHS (4.8\% in the InCHIANTI Study, 6\% in the CHS). In the other three studies, the prevalence of this criterion differed up to fourfold.

Data from the Health, Aging and Body Composition (ABC) Study [13] and the Survey in Europe on Nutrition and the Elderly: a Concerted Action (SENECA) [14], which were both large longitudinal studies, provided insight into the prevalence of weight loss in populations of community-living elderly persons. In the Health ABC Study, the subgroup with the greatest weight loss was 295 black men who lost $0.99 \pm 4.67 \mathrm{~kg}$ over a period of 3 years. In the SENECA study, the greatest weight loss was documented for Spanish men with a mean of $-4.2 \mathrm{~kg}$ over the course of a decade. These data implicate that Fried and co-workers chose a substantial cutoff for evaluating weight loss in the context of their frailty definition, resulting in a low prevalence of this criterion in a community-dwelling population. In summary, weight loss has to be regarded as a very valuable prognostic parameter for unfavorable health outcomes. However, considering the rather strict cutoffs used by Fried and co-workers, this criterion might have a low sensitivity in communitydwelling elderly persons.

In our cohort of study participants, $20 \%$ met the weakness criterion. Fried and co-workers used the measurement of handgrip strength to evaluate this criterion. In the WHAS, the InCHIANTI Study, the MrOS and the SHARE, very similar definitions of weakness were applied, resulting in similar prevalence rates across the different study populations. In the WHAS and the MrOS, the same methodology and identical cutoffs were used. In the InCHIANTI Study, the authors applied the same methodology, but used cutoffs (lowest quintile) based on data from the InCHIANTI cohort. In the SHARE Study, the investigators used the cutoffs proposed by Fried and co-workers. Nevertheless, while in the CHS the greater mean value of three measurements of each hand was the relevant parameter to evaluate this criterion, the highest value of 4 handgrip strength measurements (2 from each hand) was used in the Share Study. 
In our cohort, the most prevalent Fried criterion was exhaustion (24\%). A strong relationship between subjective fatigue and functional impairment has been shown in older people [15], which might be interpreted as evidence supporting this Fried criterion.

With regard to this criterion, significant differences are found in the four aforementioned studies. It is therefore not surprising that the respective prevalence rates differed significantly. In the WHAS, exhaustion was assumed to be present if any of the following applied: (a) a low usual energy level ( $\leq 3$, range $0-10)$; (b) felt unusually tired in the last month, or (c) felt unusually weak in the past month. The InCHIANTI Study applied the original Fried definition of this criterion. In the MrOS, a question taken from the Medical Outcomes Survey Short Form, a 12-item questionnaire, was used: 'How much of the time during the past 4 weeks did you have a lot of energy?' Responses such as 'a little' or 'none of the time' were considered to indicate exhaustion. In the SHARE Study, the exhaustion evaluation was based on the following question, 'In the last month, have you had too little energy to do things you wanted to do?'

In our study cohort, $14 \%$ of the study participants who were positive for this criterion scored 6 points or more on the Geriatric Depression Scale (GDS), indicating that they would have been considered to be depressive. Here a relevant interrelationship between a psychological disease state and the frailty definition by Fried is highlighted. A close relationship between depression and exhaustion, or more broadly fatigue, has been recognized before, especially among cancer patients [16-19]. In our opinion, the validity of this criterion must therefore be debated because the Fried definition aims primarily at a physical and not at a psychological phenotype of frailty.

Commenting extensively on the different definitions of fatigue is beyond the scope of this paper. In principle, 'peripheral' fatigue is distinguished from 'central' fatigue. According to a recent description [20], peripheral fatigue involves the end organ, i.e. muscle, while central fatigue is considered to be a failure to initiate and/or sustain attentional tasks ('mental fatigue') and physical activities ('physical fatigue') requiring self-motivation as opposed to external stimulation. Although there are several validated assessment tools for fatigue based on selfreport, a perfect tool does not yet exist. In the context of a physical phenotype of frailty, such as that proposed by Fried, it might be advisable to use a tool that assesses fatigue in relation to the level of activity. For example, the Mobility-Tiredness Scale by Schultz-Larsen and Avlund [21], which evaluates self-reported tiredness in daily ac- tivities, is a simple tool that predicts the onset of disability, walking limitations, use of health and social services, decline in physical activity and mortality. Bautmans et al. [22] showed a significant association of fatigue resistance, which may be considered an inverse equivalent of exhaustion, with self-perceived tiredness. Fatigue resistance was measured by squeezing the bulb of a vigorimeter as hard as possible, and maintaining this maximum pressure for as long as possible. These methods are valuable alternatives to evaluate exhaustion, which can be applied in a practical manner without overtly interfering with depression.

The authors of this study found a rather low prevalence of slowness (8\%). With regard to this criterion, again rather diverse definitions were applied by the authors of the four aforementioned studies. Fried et al. [7] used cutoffs for usual gait speed between 0.65 and 0.76 $\mathrm{m} / \mathrm{s}$, depending on body height. The WHAS and the InCHIANTI Study applied the same cutoffs as Fried, albeit using a walking distance of $4 \mathrm{~m}$ rather than $15 \mathrm{ft}$. In the MrOS, the adjusted cutoffs for gait speed was set at $1.06 \mathrm{~m} / \mathrm{s}$ for a 6 -meter walking test. Since previous gait speed analysis among participants above the age of $75 \mathrm{had}$ shown a close relationship between low gait speed and a positive answer to the following question 'Because of a health problem, do you have difficulty [expected to last more than 3 months] walking $100 \mathrm{~m}$ or climbing one flight of stairs without resting?', the SHARE Study applied this question to evaluate slowness.

Cutoffs for usual gait speed that correspond to adverse health outcomes vary significantly in the scientific literature. In the Short Physical Performance Battery developed by Guralnik et al. [23], the cutoff for achieving the full score in the gait speed component was set at $0.84 \mathrm{~m} / \mathrm{s}$. In the MrOS, as already mentioned, the cutoffs were set between 0.96 and $1.06 \mathrm{~m} / \mathrm{s}$. Cesari et al. [24] observed that a usual gait speed cutoff at $1 \mathrm{~m} / \mathrm{s}$ correlated with adverse health-related outcomes in relatively healthy older people. A review article on gait speed protocols was published recently [25]. The authors found a difference between static and dynamic protocols, which was not significant, indicating the necessity of a precise definition.

A practical example of the relevance of gait speed is the velocity considered the lower limit for safely crossing a signaled zebra crossing, which is $1.22 \mathrm{~m} / \mathrm{s}$ in the United States and $1.07 \mathrm{~m} / \mathrm{s}$ in the United Kingdom [26, 27]. In Germany, $1.2 \mathrm{~m} / \mathrm{s}$ is used as a cutoff point for safely crossing signaled zebra crossings [28]. Compared with these cutoffs, those established by Fried appear rather low. 
The last of the Fried criteria to be discussed is low activity. In our cohort, only $2 \%$ fulfilled this criterion. The original cutoffs were set at $270 \mathrm{kcal} /$ week for women and $383 \mathrm{kcal} /$ week for men. In women, for example, this corresponds to less than $90 \mathrm{~min}$ walking/week or $13 \mathrm{~min} /$ day only. The authors of the WHAS used the same approach as Fried, but selected only 6 activities from the original 62 of the MLTPAQ. They also reduced the cutoff for low physical activity to $90 \mathrm{kcal} /$ week. In the InCHIANTI Study, the evaluation was based on a self-report of daily physical activity in the year before the interview. Based on this self-report, the study participants were assigned to 1 of 3 categories. The lowest category, which was equal to less than $1 \mathrm{~h}$ of physical activity/week, was considered to signal frailty.

The MrOS used the Physical Activity Scale for the Elderly to evaluate this criterion. The chosen cutoff was a score of $<89.6$ (range $0-486$ ). Since this score is not transferable to kilocalories per week, it cannot be compared directly to the cutoffs established by Fried. In the SHARE Study, the participants were asked 'How often do you engage in activities that require a low or moderate level of energy such as gardening, cleaning the car, or going for a walk?' The low activity criterion was fulfilled if they responded 'one to three times a month' or 'hardly ever or never'.

In our study cohort, only 3 activities (walking, biking, swimming) from the 18 activities evaluated by Fried were relevant for the assessment of physical activity. In the original MLTPAQ, 62 different leisure time activities are assessed. This could indicate that the MLTPAQ may not be regarded as an adequate assessment tool to measure physical activity in older persons.

\section{Conclusions}

With regard to the scientific literature, the original frailty criteria established by Fried et al. [7] apply distinctively strict cutoffs for weight loss and gait speed, which contributed to a low prevalence of prefrailty and frailty among community-living older persons screened for our study. As the exhaustion criterion relies on two items of an assessment tool for depression (CES-D scale), significant overlap with this mental disease was shown in a relevant number of cases. In our opinion depression should be ruled out before an older individual is considered positive for the exhaustion criterion of Fried, as this definition aims at a physical and not a psychological phenotype of frailty. The validity of this criterion in its current form may therefore be debated. As only very few activities of the MLTPAQ were performed by older individuals, this tool may not adequately identify the level of physical activity in an older population. In addition it may be expedient to develop specific cutoffs for a European population. A prospective European multicenter study will be necessary to develop those adapted cutoffs and validate the modifications we have suggested above. In our opinion, this strategy may lead to increased awareness and the relevance of the frailty syndrome among geriatricians.

\section{Acknowledgement}

Michael Drey and Jürgen M. Bauer were supported by a Forschungskolleg Geriatrie grant from the Robert Bosch Foundation, Stuttgart, Germany.

\section{References}

1 Walston J, Hadley EC, Ferrucci L, Guralnik JM, Newman AB, Studenski SA, Ershler WB, Harris T, Fried LP: Research agenda for frailty in older adults: toward a better understanding of physiology and etiology: summary from the American Geriatrics Society/ National Institute on Aging Research Conference on Frailty in Older Adults. J Am Geriatr Soc 2006;54:991-1001.

2 Lang PO, Michel JP, Zekry D: Frailty syndrome: a transitional state in a dynamic process. Gerontology 2009;55:539-549.
3 Bergman H, Ferrucci L, Guralnik J, Hogan DB, Hummel S, Karunananthan S, Wolfson C: Frailty: an emerging research and clinical paradigm-issues and controversies. J Gerontol A Biol Sci Med Sci 2007;62:731-737.

4 Abellan van Kan G, Rolland Y, Bergman H, Morley JE, Kritchevsky SB, Vellas B: The I.A.N.A Task Force on frailty assessment of older people in clinical practice. J Nutr Health Aging 2008;12:29-37.

5 Rockwood K, Song X, MacKnight C, McDowell I, Hebert R, Hogan DB: A brief clinical instrument to classify frailty in elderly people. Lancet 1999;353:205-206.
6 Rockwood K, Song X, MacKnight C, Bergman H, Hogan DB, McDowell I, Mitnitski A: A global clinical measure of fitness and frailty in elderly people. CMAJ 2005;173:489495.

7 Fried LP, Tangen CM, Walston J, Newman AB, Hirsch C, Gottdiener J, Seeman T, Tracy R, Kop WJ, Burke G, McBurnie MA, Cardiovascular Health Study Collaborative Research Group: Frailty in older adults: evidence for a phenotype. J Gerontol A Biol Sci Med Sci 2001;56:M146-M156. 
8 Bandeen-Roche K, Xue QL, Ferrucci L, Walston J, Guralnik JM, Chaves P, Zeger SL, Fried LP: Phenotype of frailty: characterization in the women's health and aging studies. J Gerontol A Biol Sci Med Sci 2006;61:262266.

9 Ble A, Cherubini A, Volpato S, Bartali B, Walston JD, Windham BG, Bandinelli S, Lauretani F, Guralnik JM, Ferrucci L: Lower plasma vitamin E levels are associated with the frailty syndrome: the InCHIANTI Study. J Gerontol A Biol Sci Med Sci 2006;61:278283.

10 Cawthon PM, Marshall LM, Michael Y, Dam TT, Ensrud KE, Barrett-Connor E, Orwoll ES, Osteoporotic Fractures in Men Research Group: Frailty in older men: prevalence, progression, and relationship with mortality. J Am Geriatr Soc 2007;55:1216-1223.

11 Santos-Eggimann B, Cuénoud P, Spagnoli J, Junod J: Prevalence of frailty in middle-aged and older community-dwelling Europeans living in 10 countries. J Gerontol A Biol Sci Med Sci 2009;64:675-681.

12 Ferrucci L, Guralnik JM, Studenski S, Fried LP, Cutler GB Jr, Walston JD, Interventions on Frailty Working Group: Designing randomized, controlled trials aimed at preventing or delaying functional decline and disability in frail, older persons: a consensus report. J Am Geriatr Soc 2004;52:625-634.

13 Goodpaster BH, Park SW, Harris TB, Kritchevsky SB, Nevitt M, Schwartz AV, Simonsick EM, Tylavsky FA, Visser M, Newman AB: The loss of skeletal muscle strength, mass, and quality in older adults: the health, aging and body composition study. J Gerontol A Biol Sci Med Sci 2006;61:1059-1064.
14 de Groot LC, Verheijden MW, de Henauw S, Schroll M, van Staveren WA, SENECA Investigators: Lifestyle, nutritional status, health, and mortality in elderly people across Europe: a review of the longitudinal results of the SENECA study. J Gerontol A Biol Sci Med Sci 2004;59:1277-1284.

15 Vestergaard S, Nayfield SG, Patel KV, Eldadah B, Cesari M, Ferrucci L, Ceresini G, Guralnik JM: Fatigue in a representative population of older persons and its association with functional impairment, functional limitation, and disability. J Gerontol A Biol Sci Med Sci 2009;64:76-82.

16 Bower JE, Ganz PA, Desmond KA, Bernaards C, Rowland JH, Meyerowitz BE, Belin TR: Fatigue in long-term breast carcinoma survivors: a longitudinal investigation. Cancer 2006;106:751-758.

17 Ganz PA, Bower JE: Cancer related fatigue: a focus on breast cancer and Hodgkin's disease survivors. Acta Oncol 2007;46:474-479.

18 Liao S, Ferrell BA: Fatigue in an older population. J Am Geriatr Soc 2000;48:426-430.

19 Theou O, Jones GR, Overend TJ, Kloseck M, Vandervoort AA: An exploration of the association between frailty and muscle fatigue. Appl Physiol Nutr Metab 2008;33:651-665.

20 Chaudhuri A, Behan PO: Fatigue and basal ganglia. J Neurol Sci 2000;179:34-42.

21 Schultz-Larsen K, Avlund K: Tiredness in daily activities: a subjective measure for the identification of frailty among non-disabled community-living older adults. Arch Gerontol Geriatr 2007;44:83-93.

22 Bautmans I, Njemini R, Predom H, Lemper JC, Mets T: Muscle endurance in elderly nursing home residents is related to fatigue perception, mobility, and circulating tumor necrosis factor-alpha, interleukin-6, and heat shock protein 70. J Am Geriatr Soc 2008; 56:389-396.
23 Guralnik JM, Ferrucci L, Pieper CF, Leveille SG, Markides KS, Ostir GV, Studenski S, Berkman LF, Wallace RB: Lower extremity function and subsequent disability: consistency across studies, predictive models, and value of gait speed alone compared with the short physical performance battery. J Gerontol A Biol Sci Med Sci 2000;55:M221-M231.

24 Cesari M, Kritchevsky SB, Penninx BW, Nicklas BJ, Simonsick EM, Newman AB, Tylavsky FA, Brach JS, Satterfield S, Bauer DC, Visser M, Rubin SM, Harris TB, Pahor M: Prognostic value of usual gait speed in wellfunctioning older people - results from the Health, Aging and Body Composition Study. J Am Geriatr Soc 2005;53:1675-1680.

25 Graham JE, Ostir GV, Kuo YF, Fisher SR, Ottenbacher KJ: Relationship between test methodology and mean velocity in timed walk tests: a review. Arch Phys Med Rehabil 2008;89:865-872.

26 Rantanen T, Guralnik JM, Izmirlian G, Williamson JD, Simonsick EM, Ferrucci L, Fried LP: Association of muscle strength with maximum walking speed in disabled older women. Am J Phys Med Rehabil 1998;77: 299-305.

27 Moseley AM, Lanzarone S, Bosman JM, van Loo MA, de Bie RA, Hassett L, Caplan B: Ecological validity of walking speed assessment after traumatic brain injury: a pilot study. J Head Trauma Rehabil 2004;19:341348.

28 Boltze M, Friedrich B: Innovation in der Lichtsignalsteuerung - Die Neufassung der Richtlinien für Lichtsignalanlagen (RiLSA); in Tagungsband (CD-ROM) des Deutschen Strassen- und Verkehrskongresses, 27.-29. Sept, Karlsruhe: Strassenverkehrstechnik. Bonn, FGSV, 2007, vol 4, pp 192-197. 